\title{
Article
}

\section{Making it 'APP'en: service user feedback: developing and implementing a service user APP: reflections from Northern Ireland, England and Scotland}

Westwood, Joanne Louise, Dill, Katharine, Campbell, Anne and Shaw, Alaine

Available at http://clok.uclan.ac.uk/23382/

Westwood, Joanne Louise ORCID: 0000-0002-7560-1391, Dill, Katharine, Campbell, Anne and Shaw, Alaine (2017) Making it 'APP'en: service user feedback: developing and implementing a service user APP: reflections from Northern Ireland, England and Scotland. Social Work Education, 36 (8). pp. 855-868. ISSN 0261-5479

It is advisable to refer to the publisher's version if you intend to cite from the work. http://dx.doi.org/10.1080/02615479.2017.1360857

For more information about UCLan's research in this area go to http://www.uclan.ac.uk/researchgroups/ and search for <name of research Group>.

For information about Research generally at UCLan please go to http://www.uclan.ac.uk/research/

All outputs in CLoK are protected by Intellectual Property Rights law, including Copyright law. Copyright, IPR and Moral Rights for the works on this site are retained by the individual authors and/or other copyright owners. Terms and conditions for use of this material are defined in the policies page. 



\section{Introduction}

4 Local authorities, community and voluntary sector organisations providing services are

5

6

Service user perspectives in social work practice and education

The voice and perspective of service users is essential to ensure the implementation of quality, effective and compassionate social work service. Efforts to ensure service user perspectives inform social work are included in recent UK policy and legislation (Hernandez, Robson and Sampson 2010). Service user led movements have promoted participatory and 'ground up' approaches which move beyond consultation. Campaigning groups and organisations argue that their perspectives must become central to building and supporting social work educational knowledge, organisational best practice. Capturing service user perspectives also addresses the needs and rights of socially and politically marginalised groups (Postle and Beresford 2007).

Student social workers are well-versed in engaging with and learning from service user perspectives. Students graduate from social work education programmes in the UK with the rich and rewarding realisation that the service user is the pivotal link for learning about the 
essential social work skills to carry out a clear, empathic and compassionate interaction with a service user. Learning from the service user perspective may diminish once a student enters the social work workforce as service delivery and resource management is prioritised. However it is incumbent upon social workers to practice with respect and courtesy and keep service users informed of what is happening. In addition social workers must adhere to relevant codes of conduct as stipulated by the Health and Care Professions Council (HCPC) in England; Scottish Social Services Council (SSSC) and the Northern Ireland Social Care Council (NISCC).

\section{There are several approaches to service user involvement in social work practice, research and} evaluation (Beresford 2002) including those which are led and designed by or in partnership with service users. Democratic models are designed and produced by service users and foreground their involvement, control and power in the processes involved and in terms of determining outcomes. Consumerist approaches adopt a customer service orientation in line with the market economy with a service improvement outcome focus. There are several methods of gathering feedback from service users in the consumerist approach including direct consultation where service users give feedback on services to the social worker, through interviews, surveys, questionnaire or through participation in focus groups. These may be internally or externally facilitated and may be for multiple purposes including inspection regimes or for service developments (Beresford 2002).

Service user evaluation using new technologies

The project aims were to firstly develop an APP which could be used by social workers to gather real time feedback from service users. Secondly our aim was to pilot the APP with different service user groups and social workers based in contrasting settings and contexts. Finally the project aims to evaluate the responses gathered using the APP and analyse the findings of the pilot using focus group methodology and report on the implementation.

The SU-APP pilot project was designed to capitalise on emerging developments in the digital landscape of social work education, framing this education as a career long process of which 
qualifying social work training is merely the beginning. The pilot implementation phase of the project where social workers using the SU-APP request service users' feedback on their experiences we envisage will facilitate an engaged, responsive and participatory process of service user involvement, as the evaluation data collected using the device starts to inform and shape services.

Advances in technology and drivers for increasing digital citizenship are pushing forward the development of new ways of consulting with, and gaining feedback from, people who use social services. Our approach to working with the three pilot sites encouraged practitioner involvement and participation given that new technologies might be resisted and/or greeted with caution in terms of their utility in improving or changing social work practice.

Traditional systems of gathering service user feedback (paper based surveys and interviews) are time consuming and may not be efficient in terms of the storage and retrieval of information provided (i.e. paper based survey data). It is important to recognise when we embrace technologies in practice and in social work education that there is a digital divide. Socio economic as well as connectivity issues impact on the use and ownership of new technologies (Cabinet Office 2014). Service users and practitioners might regard themselves as digital visitors (White and Le Cornu 2011) and lack the digital literacy required to use technologies effectively in their daily lives. However global increases in SMART phone and tablet ownership (GW1 2014) suggest that many more people are residents in the new digital landscape, whilst wider government agendas are pushing digital citizenship forward. Research also suggests that local authorities are engaging with digital technologies for example in customer services orientated aspects of their work (BDO 2012; BDO 2015).

The availability of new technologies demand that as researchers, educators and practitioners we consider how to collate service user feedback in different ways so that agencies and organisations can respond to an ongoing feedback and evaluation process which is integrated as part of their service delivery model. We also need to ensure that our approach to developing new ways of gathering evaluation and feedback from service users is compliant with the ethical and value base of social work and maintains the rights and dignity of people we work with (BASW 2012). Student social workers and those involved in their education are required to ensure that they have an understanding of how technology informs and shapes their practice, as the BASW policy states that: "social workers need to be aware of and knowledgeable about 
technological developments and understand the impact, use and advantages as well as possible ethical concerns and risks in relation to themselves, the people they are working with and their employers" (BASW 2012, p.5).

The SU-APP project draws on a knowledge mobilisation approach for moving service user knowledge into practice (authors own a) informed by social work traditions and values of engaging service user perspectives to influence practice. The project was driven forward through engagement with existing local and national agendas which seek to advance the contribution of service users to changing how services are developed and delivered. The project drew on partnerships between key actors in commissioning services who provided some funding, and access to local agencies working in social care, and a technology company with prior experience of developing apps for social work education and training. This paper now discusses the challenges, opportunities and lessons learned in developing this new instrument, observations of the project team involved in the initial development and pilot phase and highlights ways in which these challenges were navigated and addressed.

\section{New Thinking about Facilitating Service User Feedback:}

The project team aimed to develop a novel way for social workers to collate evaluation data from service users, with their informed consent, about services they had received at the end of a meeting or visit from a social worker. As social work educators in Higher Education Institutions (HEI's) and in workforce development, the project team came together to consider how best we might use SMART technologies and digital devices to facilitate the collation of service user feedback and evaluation and provide both students and practitioners with evidence of how we can use new technologies in our work. SMART devices lend themselves well to this type of approach as they have the capacity of Personal Computers (PCs) yet are small enough to be easily portable, held in one hand and combine interactive touch screen features with high resolution graphics and visuals. SMART devices are also able to host Apps (mobile software). 
1 Despite the advent of social media and increasing use of SMART technology amongst global and UK populations, APP's for social work remain in their infancy (Authors own b). Several have been developed which focus on the education of social workers and practitioners which provide information on specific social work issues. There are a small number of Apps available for social work activities, for use in both social work education and for practice covering topics including child development for different age ranges (Authors own b; Authors own c Authors own d; Authors own e), and an APP for social workers focussing on substance misuse (Authors own f), designed to provide easily accessible practice information and theory for social workers. These are free at the point of use and there is evidence to suggest that these have been used widely in practice in the UK and on an international basis ( Campbell \& Mc Colgan 2016. In addition there is an APP designed to engage students and practitioner in key ethical issues regarding the use of social media in social work practice (Singh Cooner 2013). This APP introduces practice scenarios and users respond to questions related to online behaviour in social work practice and is supplemented with an educators' workbook for use with students either in the classroom or in the field. We are also aware that there are emerging health feedback platforms such as the Patient Feedback APP (Heart of England NHS Foundation Trust 2016) and Patient Survey APP (iCheckup, LLC 2016) but at the time of writing we are not aware of an APP that has been developed to gather feedback from social work service users at the point of intervention.

\section{Development of the $S U-A P P$}

The pilot phase of this project is currently taking place in Northern Ireland, England and Scotland and involves both service users and social workers/support workers. Three organisation are involved in piloting the SU-APP, an initial assessment team in Northern Ireland, a children and families service in England and a third sector substance misuse service in Scotland. The evaluation model being used for the pilot phase is designed to document the implementation process, explore what works and what barriers or challenges workers experience in gathering data using the SU-APP. Social workers will be invited to report on the feedback they receive from service users they work with who agree to use the SU-APP. The data from the pilot phase and the evaluation will contribute towards the development of a range of feedback APPS for different service user groups and instructional materials for student social 
1 workers and practitioners to ensure that they are familiar with how these digital tools can augment their practice.

$4 \quad$ Methods

5 Developing the $S U-A P P$

6 Two members of the research team based at different universities in the UK met at a national

7 conference hosted by a UK based training and development organisation where they were

8 presenting sessions to the social work practitioner/trainer audience about the increasing use of

9 social media and digital technologies and exploring the implications of this increase for social

10 work practice. A training development lead for an LA was considering different ways in which 11 digital technologies could be utilised by practitioners and was also present at this conference.

12 This meeting led to a discussion and the design of a very simple prototype for the design of the 13 SU-APP. A fourth member of the team was recruited for her expertise and experience in designing and implementing Apps.

APP

The original idea for the SU-APP came from a practitioner in response to formal inspection reports and requirements that service users provide feedback on social work services. The emphasis in the development of the SU-APP was in terms of creating an easily accessible mobile instrument which social workers can use to gather anonymous feedback from service users about their experiences of social work interventions. The project began with an outline concept about how technologies could facilitate service user feedback at the point of social work intervention. Funding was secured in small amounts from several service provider agencies based on this early conceptual work and the project team worked with an independent technology company to design the prototype for the pilot phase.. In doing so the project team utilised digital technology and social media to maintain momentum and enthusiasm for the project which simply would not have been possible in a pre-web 2.0 world. 
1 The project team shared their work in progress at two conferences in the UK with service user and social work practitioner audiences and received helpful and critical feedback about the design features and practical issues we would need to consider for the implementation of the SU-APP as it was under development. One such audience was largely made up of service users from across the UK and the project team received encouraging feedback and questions about the practicalities of implementation which helped them to consider the realities of social workers seeking feedback and what advice and information could be provided to prepare them for this.

\section{Pilot phase: social workers use the APP to gather evaluation data}

Social workers who agreed to pilot the SU-APP reported that it would be too cumbersome for the service users to provide feedback on the social work interaction followed by service user reflection on their experience of using the SU- APP. Therefore, the social workers agreed to report on their own and the service user's perception of using the SU-APP as a means for feedback (see figure 1. below). The research team acknowledge the limits of this $3^{\text {rd }}$ party reporting in terms of who retains power in the process of the pilot, and that $3^{\text {rd }}$ party feedback from social workers, may not fully capture service user perspectives. The decision was made following discussions with the social workers involved in the pilot.

\section{Fig. 1. Feedback from social workers and service users}

\section{Design and Evaluation Method}

\section{Service user involvement:}

The SU-APP was designed in several stages. A brief paper outlining the background to the project and the proposal for the SU-APP was prepared and targeted at several local authorities and organisations. Several of these agencies expressed an interest in the SU-APP but were reluctant or unable to provide funding to go ahead with the design and pilot phase. After a period of negotiation, funding was obtained from one of the University partners from an 
innovation fund administered by the Health and Social Care Board in Northern Ireland, from one local authority in England and from a third sector agency in Scotland.

The evaluation survey which forms the basis for the SU-APP was initially designed by the project team and received feedback from a service user panel and from social workers before being piloted. The consumerist model (Beresford 2002) was adopted in the project development phase, whereby a service user panel along with the stakeholder group provided feedback on the original design. The discussion and issues raised at this panel included concerns about who would have access to the data that was collated. The research team took the concerns about anonymity and confidentiality very seriously as the intention was to gather data from service users which would not be attributable to individual social workers but contribute to service improvement at an organisation level. The project team were also concerned to ensure that individual service users who provided feedback using the SU-APP could not be identified. We acknowledge the limited service user input into the original design of the SU-APP, and the development of the project in the post pilot phase will draw on social workers providing critical feedback and evaluation from service users participating in the pilot. We also need to accept that the integrity and validity of this approach must be explored further in the post pilot phase of the project. Prior to the initiation of a next phase of the research, which will include a proposed larger study of the APPs efficacy in practice, it is intended that service users will be consulted and asked to provide their opinion on the general usability of the APP as a mechanism for feedback and any ethical issues that might arise for them as a result of its usage as part of the assessment / intervention processes.

The questions agreed upon were reviewed by members of the local authorities in two of the pilot sites who provided feedback on the overall utility of the questions proposed and recommended new questions for consideration. All questions were designed using a Likert scale format to facilitate ease of service user response and straightforward data input for later analysis. There was also discussion to ascertain if the questions being asked appeared reasonable and non-threatening and would ultimately facilitate engagement with the service user. Feedback on the questions suggested that some questions might be developed which gather qualitative data and this will be considered following the pilot. The project team had several discussions with social workers during the first phase of the pilot stage where the SUAPP was being introduced about working with resistance and the challenges to facilitating 
1 feedback in such situations. Social workers involved in the information sessions were keen to

2 work alongside service users to provide a different way of gathering feedback using this

3 technology, and regarded themselves as instrumental in facilitating feedback through the SU-

4 APP.

5

$6 \quad$ Piloting the APP: the process for social workers

7 In the initial development phase of the SU-APP, the method of collating the date was discussed

8 as follows: the social worker would hand a tablet or SMART mobile device to the service user 9 at the end of a meeting. This individual (service user) would then answer a series of questions

- Social worker opens the SU-APP and is presented with a screen to 'select question set'

- The SU-APP then shows an introduction page with some instructions/guidance

- The social worker passes the device to the service user

- The service user reads the instructions and clicks the start button.

- The service user responds to a series of 10 questions on consecutive screens which give options for responses ranging from negative to positive.

- After the last question, the service user submits the feedback using the send button

- At this point, the feedback is submitted directly to the SU-APP database accessible only by the research team and neither the social worker nor the service user can view the responses.

- The service user then closes the SU-APP which returns to the home screen

- The service user returns the device to the social worker. 
2

3

4

SU-APP questions:

The SU-APP was designed to gather quantitative data, the emphasis in terms of feedback was to gather data which focusses on issues related to communication with service users, clarity and to confirm mutual understanding of the social work objectives. The questions 1-8 are rated from strongly disagree through to strongly agree, whilst questions 9 and 10 range from very negative to very positive. The question response options shift from a negative rating upwards to a positive rating scale to ensure that feedback is based on realistic perceptions and views:

1. The social worker appeared prepared for the interview with me

2. I felt valued during my visit with my social worker

3. I was clear about what the social worker was able to do and not do

4. I felt okay about giving my views to my social worker

5. I felt listened to by my social worker

6. I was clear about the purpose of my social work interview

7. I understand the plan made with my social worker today

8. My discussion with my social worker was helpful

9. I would rate the quality of my social work experience as:

10. My experience with my social work agency was:

Information sessions for social worker involved in the SU-APP pilot

Prior to the launch of the pilot, information sessions are held with the social work /agency teams who are planning to pilot the SU-APP to discuss any questions, concerns or issues that workers may have, about their use of the SU-APP and to confirm what will happen to the data that they collect. As part of the pilot programme, service users provide feedback directly into the SU-APP during their interview with their assigned social worker as outlined above. This is designed to minimise interruption of worker/ service user interaction and reduce feedback time for service users . 
1 Social workers from family and childcare teams in a Health and Social Care Trust in Northern

2 Ireland and a Council area in England, and a third sector organisation working in drug and

3

After administration of the SU-APP there will be six focus group sessions with social workers and managers involved in the piloting of the SU-APP; two in each pilot area. Focus groups will include 6-8 social workers and managers and stakeholders who have been involved in the creation and development of the SU-APP. Social workers' feedback on the SU-APP will focus on the workers' thoughts on usability, their perceptions of services users' views on the SUAPP, the viability of the SU-APP in gauging views on practice and any barriers that hindered ease of access and usage.

\section{Data Analysis}

Responses from the three pilot sites will be sent directly to the project team and will be held in a central password protected online repository by the lead researcher. In addition, a database will be created in SPSS (a specialised computer package) and the data will be transposed directly from the online repository into SPSS, for analysis. The quantitative data will be presented pictorially through the use of graphs and descriptive statistics. In addition, the Likert data will be analysed through cross tabulations and chi square analysis to examine possible association between variables.

Data from the six qualitative focus groups, will be digitally recorded and transcribed verbatim. Qualitative data analysis will be conducted using the Burnard (1991) framework for thematic data analysis using both nominal and narrative approaches (see, for example, Lewis-Beck, et 
al 2004, Bryman 2008).Inter-rater checks on the focus group transcripts will be carried out by two members of the research team. Through this process, a range of categories will be developed, coded and reduced in order to identify emergent themes and issues and to explore the relationships between issues such as 'ease of use' and perceptions of the SU-APPs relevance to practice.

\section{Ethical Considerations}

The key ethical issue in this study is that Answering questions in front of their social worker could make service users feel somewhat uncomfortable about being open, or critical of the services that they receive or about the communication and listening skills of their social worker. For example, a service user may feel that giving negative feedback or a critique of the services provided to them may result in diminished services. . This issue of power and authority is clearly explained and identified in the study participant information form and will be reiterated by the social worker prior to and during the piloting activity. The study will also be closely monitoring how the power and authority issues are navigated between the service user, practitioner and broader organisation in the focus groups with workers after the pilot phase.

It is important that the identity of the service user is kept confidential at all times. There are a number of circumstances that may make it possible to identify the user (date, location of feedback, device type used to capture feedback). The technology company commissioned to do this work along with the project team will mitigate this risk by not revealing time stamps, locations or device types to third parties including our own research team. Timings of feedback submissions will be limited to the week of submission only and the order of records will be randomized in the delivered records. We will use device type and location of information to help review the performance of the SU-APP but this information will not be passed on to the participating organisation or practitioners involved in the pilot.

Ethical approval has been granted by Queens University Belfast (QUB) Ethics Committee, the University of Stirling Ethics Committee and the Health and Social Care Trust research 
governance committee in Northern Ireland. Two key ethical issues raised by the QUB committee were the anonymity of service users who were going to be using the SU-APP to give feedback, and the anonymity of the social workers who would be engaging with service users to solicit their feedback. In response, the SU-APP development team assured the committee that the anonymity of all parties would be protected via the specifically designed SU-APP response system and through recognised methods outlined above to protect the anonymity of research participants.

\section{Discussion}

There are some key learning point which have emerged during the development phase of this project. The project team were aware at the outset that this was a new and innovative method to gather service user feedback and it would be challenging to maintain the initial enthusiasm and momentum and convince stakeholders of the value of trying this new approach.

It is clear that practitioners bring a wealth of experience to research projects such as these and innovative ideas should be supported for these to be implemented. The academic world can sometimes seem to be removed from these creative and on-the-ground strategies. It is important that practitioners feed social work and social care ideas from experience into research and development agendas in HEIs, so that student social workers can develop their understanding of creativity and innovation opportunities in practice, whilst also learning about different approaches to seeking and responding to service user feedback and evaluation.

Building university-practice partnerships takes time, energy and commitment. Academic institutions and funding regimes do not always lend themselves well to supporting innovative projects such as this. The project team relied heavily on a small number of key people in organisations and in the HEIs to build these partnerships. Whilst the project team was able to secure small funds from several stakeholders, there were several processes and systems to navigate all with their own criteria and regulations which made access to the funding difficult and time consuming. Support from HEI's needs to be multi-faceted and include assistance in helping project teams navigate change and deal with ethical issues especially in projects such as this where new technologies are being used in practice with service users. 
2 Finding the right innovation partner was crucial. The technology company that the research team worked with were flexible and creative. Their experience and understanding of the sector and what the project team were trying to achieve was really valuable. As discussed earlier in the development phase service users consulted about the SU-APP questions raised important concerns about what happens to the information that they provide and how this is kept confidential and social workers shared these concerns. The SU-APP developer's contribution to the discussions about the implementation of the SU-APP informed the ethics application and brought ideas which were incorporated into the finished instrument. One of the primary challenges of working within the field of online technology and innovation is securing a partner who has knowledge of the social work sector and concomitant expertise in APP development (Authors own g). In the partnership that became established there was a reciprocally engaging and effective collaboration which was based on a fluid and responsive working relationship. The APP developers had knowledge of the social care sector and was informed about the importance of user feedback through development of similar applications in medical and social care. Likewise, the social work education and practice based content development team had a prior experience of working within online innovation, social media and APP development. What followed was a symbiotic and exciting exchange of ideas between APP developers and the creative content team. Indeed, the development of the SU-APP became an organic process that reacted to rapidly developing ideas often on a daily basis throughout the working collaboration.

The project aimed to test the SU- APP as a mechanism for obtaining service user feedback primarily from the perceptions of the social workers. By default, the service user would be providing feedback on the service but not necessarily the SU -APP itself. The pilot project also considered whether an APP format would better facilitate the gathering of feedback and encourage the service user to provide information at the point of contact. As referred to above, social workers at the planning stage highlighted that obtaining service user feedback via the SU-APP might prove to be an onerous task and that service users would likely feel overwhelmed if they participated in a second stage of feedback to gauge their perceptions of using the APP. Consequently, it was agreed that social workers would report on service user perceptions of using the APP through the worker focus groups. This limitation of the pilot 
1 project will be addressed in the follow up main study which will include service use feedback through all elements of the design and the delivery of the study.

\section{Limitations of SU-APP Pilot}

5 We have learned from our failure to build towards success (Pattoni, 2013). Trying something new will always have its challenges. Where we wish to inspire others is in the steadfast belief that a good idea for social work practice will earn its place in time (Fang, Mishna, \& Hons, 2014; Mishna, Bogo, Root, \& Khoury-kassabri, 2012). The challenge with the design and development of the SU-APP was the real or imagined perception that the project team would always get the design and implementation of this tool right from the start. In our initial naïve desire to create a new platform for synthesizing and integrating service user feedback, we hit some 'bumps' along the way. At the inception of the idea, our team worked together across virtual interfaces to meet these challenges on an almost weekly basis. These challenges included: asking the right questions and indeed, were there ever any of the right questions, how do we engage service users in designing the APP, and how could we encourage practitioners to agree to using an instrument that may say negative things about their practice? The information sessions to date have contributed somewhat to these issues and we are now in a position to learn from the pilot phase.

In the design phase of the SU-APP we would have liked to have a more engaged service user feedback (Authors own h). Our several attempts to facilitate this were not successful and ultimately we were only able to facilitate limited engagement with service user feedback at the beginning. As we go forward into phase two of our design with the involvement of service users, we realize that future iterations of the SU- APP must address how the instrument can facilitate diverse needs such as non-English speakers, service users with sight impairments and other issues and themes raised by practitioners. Perhaps the success in our story is that we embraced the imperfections of the SU-APP design platform to create an instrument that will continue to evolve in its iteration.

Whilst the project team have been driving this project forward, it is important to reiterate that this is a pilot project and the SU-APP has been designed to be tested out rather than be the finished item. The next phase of work will reveal what additional development needs to be 
carried out before we can formally launch it. The project team accept that the SU-APP in its current iteration is not perfect, but it is the starting point. Throughout the process of design and development the project team have operated from a shared perspective of being explicit about what the SU-APP is designed to do, and importantly what it cannot and should not do in terms of privacy, confidentiality and anonymity. These perspectives and the value base of the project team rooted in social work have enabled a sharing and exchange of knowledge. The project team was always clear with key stakeholders that the idea is evolving and through the piloting of the project, the feedback tool can be shaped in response to the evaluation for future implementation and application.

Capturing the service user experience is a core facet of the pedagogical approach to social work education and practice in the UK. Through the development of an APP for service users we can further emphasise the central message of involving service users in a truly participatory manner. We can indicate to students how this is operationalised in practice through the development of the current project and others which consider service user involvement in education and practice on a number of levels. Students begin their educational journey by receiving feedback from service users; the SU-APP provides an opportunity to allow for continuous feedback engendering a culture which positions seeking feedback as the norm for practice.

Online learning tools, applications and the use of technological innovations are becoming a central part of the social work learning process and social work educators must be aware of the rapidly changing patterns of use of innovative online applications and systems amongst our students, practitioners and service users. The current project serves to reinforce this linking of technology, practice and education. It also highlights to social work students how we must look to technological advancement to further include service users and increase their awareness and knowledge of the paradigm shift from paper based assessment and evaluation to online methods of data gathering and analysis. 


\section{References}

2 Authors own a

3 Authors own b

$4 \quad$ Authors own c

5 Authors own d

6 Authors own e

7 Authors own $\mathrm{f}$

8 Authors own g

9 Authors own h

BASW (2012) BASW Social Media Policy. http://cdn.basw.co.uk/upload/basw 34634-1.pdf

BDO (2012) from housing and litter to Facebook and Twitter

http://www.bdo.co.uk/ data/assets/pdf_file/0008/186524/BDO_Local_Government_Team_-

_Updating_your_status_social_media_report.pdf

BDO (2015) What's happening in Local Government Social Media: a review of social media usage in UK local government https://www.bdo.co.uk/getattachment/70a28fb3-2b3a-4baf9469-a982158d3a90/attachment.aspx

Beresford, P. (2013). From "other" to involved: user involvement in research: an emerging paradigm. Nordic Social Work Research, 3 (February 2015), 139-148. http://doi.org/10.1080/2156857X.2013.835138

22 Beresford, P. (2002). User Involvement in Research and Evaluation: Liberation or

23 Regulation? Social Policy and Society, 1, pp 95-105 doi: 10.1017/S1474746402000222 
1 Cabinet Office /Government Digital Service (2014) Digital Government Digital Inclusion

2 Strategy https://www.gov.uk/government/publications/government-digital-inclusion-

3

4

strategy/government-digital-inclusion-strategy

Campbell, A., \& McColgan, M. (2016). Making Social Work Education App'ier: The Process of Developing Information-based Apps for Social Work Education and Practice. Social Work Education: The International Journal. 10.1080/02615479.2015.1130805

APP

Fang, L., Mishna, F., Zhang, V.F., Van Wert, M. \& Bogo, M. (2014) Social Media and Social Work Education: Understanding and Dealing with the New Digital World, Social Work in Health Care, 53:9, 800-814.GWI (2014) Global Device Summary Q3.Available athttps://www.globalwebindex.net/

Health Care Professions Council (2012.) http://www.hcpc-uk.co.uk/

Heart of England NHS Foundation Trust (2016) Patient Feedback APP

http://www.heartofengland.nhs.uk/patient-feedback-APP/

Hernandez, L. Robson, P., and Sampson, A. (2010) Towards Integrated

Participation: Involving Seldom Heard Users of Social Care Services. British Journal of Social Work 40, pp 714-736 doi:10.1093/bjsw/bcn118

iCheckup, LCC (2015) Patient Survey https://itunes.apple.com/gb/APP/patient$\underline{\text { survey/id605066833? } \mathrm{mt}=8}$

Lewis-Beck, M. S., Bryman, A., \& Liao, T. F. (Eds.). (2004). The Sage encyclopaedia of social science research methods (3rd ed.). Thousand Oaks, California: Sage

Mishna, F., Bogo, M., Root, J., \& Khoury-kassabri, J. S. M. (2012). “It just crept in” "”: The Digital Age and Implications for Social Work Practice, pp.277-286. http://doi.org/10.1007/s10615-012-0383-4

Northern Ireland Social Care Council (2016) http://www.niscc.info/ 
1 Pattoni, L. (2013). Don’t we Learn More from Failure? Retrieved May 15, 2016, from

2 http://www.iriss.org.uk/resources/iriss-failure

3 Postle, K., and Beresford, P., (2007) Capacity Building and the Reconception of Political

4 Participation: A Role for Social Care Workers? British Journal of Social Work (2007) 37,

5 pp.143-158 http://doi.org/10.1093/bjsw/bch330

6

$7 \quad$ Scottish Social Services Council (2016) http://www.sssc.uk.com/

8 Singh Cooner, T. (2013) Social Work Social Media APP

9 https://itunes.apple.com/gb/APP/social-work-social-media/id656114442?mt=8

11 Smith, M., Gallagher, M., Wosu, H., Stewart, J., Cree, V.E. Hunter, S., Evans, S.,

12 Montgomery, C., Holiday S., and Wilkinson, H., (2012) Engaging with Involuntary Service

13 Users in Social Work: Findings from a Knowledge Exchange Project. British Journal of

14 Social Work 42, pp. 1460-1477 Retrieved from

15 http://bjsw.oxfordjournals.org/cgi/content/long/bcr162v1 